DISCUSSION/SIGNIFICANCE OF FINDINGS: This trial proposes a novel collaborative care approach for opioid weaning using proven, easy-to-deliver positive psychology tools for pain management that, if successful, could be implemented broadly in many clinics struggling to safely reduce opioid prescribing.

73936

Developing a Patient-Rated Outcome Measure of Alcohol and Drug Craving: A Systematic Review

Angela M. Haeny, $\mathrm{PhD}^{1}$, Meghan Morean, $\mathrm{PhD}^{1}$, Kelly S. DeMartini, $\mathrm{PhD}^{1}$, Melissa Funaro, MLS, MS ${ }^{2}$ and Stephanie S. O'Malley, $\mathrm{PhD}^{1}$ ${ }^{1}$ Yale School of Medicine and ${ }^{2}$ Yale University Medical Library

ABSTRACT IMPACT: The findings from this study will inform the development of an FDA-approved patient-rated outcome measure of drug and alcohol craving that can be used in clinical trials aimed at developing or testing effective treatments for substance use disorder. OBJECTIVES/GOALS: Craving is a potential target of investigative medications to reduce drug use due to the strong link between craving and drug use. We will identify all existing craving measures as the first step for developing an FDA-approved patient-rated outcome measure for use in clinical trials. METHODS/STUDY POPULATION: Following PRISMA guidelines, we will update Rosenberg's (2009) craving review by conducting a systematic review of all existing published and unpublished measures of craving for alcohol, nicotine, cannabis, opioid, and stimulant use. Electronic database (i.e., Ovid MEDLINE, Embase, PsycINFO, Web of Science, Cochrane), forward, backward, and author searches will be conducted. We will also request unpublished craving measures on major listservs (e.g., Research Society on Alcoholism, the Collaborative Perspectives on Addiction, and the College on Problems of Drug Dependence). All papers included in Rosenberg's (2009) review through September 2020 will be included. RESULTS/ANTICIPATED RESULTS: The findings from this review will provide a comprehensive summary of the construct of craving and its hypothesized and tested domains. This review will elucidate whether the literature suggests there are components of craving unique to alcohol, nicotine, cannabis, opioid, and/or stimulant use, and whether there are key elements of craving common across the disorders. Therefore, these findings will inform whether a single patient-rated outcome measure of craving can be developed for use across substances or if unique patient-rated outcome measures of craving need to be developed for each substance. DISCUSSION/SIGNIFICANCE OF FINDINGS: While many different measures of craving exist, none have gone through the developmental steps required to qualify as an FDA-approved patient-rated outcome measure on which drug treatment labeling can be based. Completing this systematic review is the first step in this process.

77286

Intravital microscopy in the study of the tumor vasculature of patients with peritoneal carcinomatosis Emmanuel Gabriel ${ }^{1}$, Minhyung Kim², Daniel Fisher², Catherine Mangum $^{1}$, Kristopher Attwood ${ }^{2}$, Wenyan $\mathrm{Ji}^{2}$, Debabrata Mukhopadhyay ${ }^{1}$, Sanjay Bagaria ${ }^{1}$, Matthew Robertson ${ }^{1}$, Tri Dinh ${ }^{1}$, Keith Knutson ${ }^{1}$, Joseph Skitzki ${ }^{2}$ and Michael Wallace ${ }^{1}$

${ }^{1}$ Mayo Clinic Florida and ${ }^{2}$ Roswell Park

ABSTRACT IMPACT: Investigation of tumor-associated blood vessels may serve as an imaging biomarker of response to systemic therapy and cancer outcomes. OBJECTIVES/GOALS: Aberrancies in the tumor microvasculature limit the systemic delivery of anticancer agents, which impedes tumor response. Using human intravital microscopy (HIVM), we hypothesized that HIVM would be feasible in patients with peritoneal carcinomatosis $(\mathrm{PC})$ and generate clinical utility. METHODS/STUDY POPULATION: During cytoreductive surgery with hyperthermic intraperitoneal chemotherapy for PC, HIVM was performed in both tumor and non-tumor areas. The primary outcome was HIVM feasibility to measure vessel characteristics. We secondarily evaluated associations between HIVM vessel characteristics and oncologic outcomes (RECIST response to neoadjuvant therapy and disease-specific survival). RESULTS/ ANTICIPATED RESULTS: Thirty patients with PC were enrolled. Nineteen patients $(63.3 \%)$ received neoadjuvant therapy. HIVM was feasible in all patients. Compared to non-tumor (control) areas, PC areas had a lower density of functional vessels, higher proportion of non-functional vessels, smaller lumenal diameters, and lower blood flow velocity. Qualitative differences in these vessel characteristics were observed among patients who had partial response, stable disease, or progressive disease after receiving neoadjuvant therapy. However, no statistically significant relationships were found between HIVM vessel characteristics and oncologic outcomes. DISCUSSION/SIGNIFICANCE OF FINDINGS: These novel findings comprise the first-in-human, real-time evidence of the microscopic differences between normal and tumor-associated vessels and form the basis for our larger, ongoing clinical trial appropriately powered to determine the clinical utility of HIVM (NCT03823144).

93096

\section{Does gender matter? Gender differences in the relationship between resting-state functional connectivity and emotion regulation in alcohol use disorder.}

Kai Xuan Nyoi ${ }^{1}$, Emily Koithan ${ }^{1}$, Timothy Hendrickson ${ }^{2}$, Hannah Verdoorn ${ }^{1}$, Casey Gilmore ${ }^{3}$, Bryon O. Mueller ${ }^{1}$, Matt Kushner ${ }^{1}$, Kelvin O. Lim $^{1,3}$ and Jazmin Camchong ${ }^{1}$

${ }^{1}$ University of Minnesota Department of Psychiatry and Behavioral Sciences, ${ }^{2}$ University of Minnesota Informatics Institute and ${ }^{3}$ Minneapolis VA Health Care System

ABSTRACT IMPACT: Our research has the potential to impact human health by identifying gender specific neural markers of emotion regulation in alcohol use disorder. OBJECTIVES/GOALS: Emotion dysregulation is known to be mediated by altered functional organization of the limbic system in addiction. This preliminary study sought to identify gender effects in the association between emotion regulation and resting-state functional connectivity (rsFC) of a negative affect network. METHODS/STUDY POPULATION: 55 individuals receiving treatment for alcohol use disorder ( $\sim 2$ weeks of abstinence) were recruited for this study and included in this analysis $(\mathrm{N}=55$; Age: $\mathrm{M}=41.78, \mathrm{SD}=10.66 ; 21$ females). RsFC within a network involved in the withdrawal/negative affect stage of addiction and Personality Inventory for DSM-5 (PID5) metrics were collected from all participants. RsFC data were preprocessed using the Human Connectome Project pipelines. Correlations between (a) rsFC within the withdrawal/negative affect network and the (b) scores of the negative affect subscale of the PID5 instrument were conducted for each gender separately. RESULTS/ ANTICIPATED RESULTS: Independent samples t-test showed a statistically significant gender difference in the PID-5 negative affect 